\title{
An Exact Impedance Control of DC Motors Using Casimir Function
}

\author{
Satoru Sakai \\ Shinshu University \\ Japan
}

\section{Introduction}

This chapter gives a new and exact impedance control of DC motor. From Hogan's original work, the control input for impedance control is torque input since the impedance control is designed for Lagrangian systems. However, in actual situation, there exist dynamics between the torque and control input and this dynamics can be dominant in certain scale. In such situation, if we neglect the dynamics or try to cancel the dynamcis, the standard impedance control can lose the stability or the control performance at least.

To overcome this problem, we need an new impedance control which takes the dynamics into account wihtout canceling any dynamics. In this chapter we give a solution for this problem by focusing on Casimir function which is rarely used in the conventional robotics.

The rest of this chapter is organized as follwows. In Section 2, we give a new model of DC motor with dynamics between the torque and control input. In Section 3, we propose a new impedance control which is based on Casimir function. Casimir function is one of the properties of port-Hamiltonian systems. In Section 4, we confirm the proposed method in numerical simulation and we conclude this chapter in Section 5.

\section{Modeling}

Let us start from a well-known model of DC motor:

$$
\left[\begin{array}{c}
\dot{\theta} \\
\dot{\omega} \\
\dot{i}
\end{array}\right]=\left[\begin{array}{rrr}
0 & \frac{1}{J} & 0 \\
0 & 0 & K \\
0 & -\frac{K}{L} & -\frac{R}{L}
\end{array}\right]\left[\begin{array}{c}
\theta \\
\omega \\
i
\end{array}\right]+\left[\begin{array}{c}
0 \\
0 \\
\frac{1}{L}
\end{array}\right] v
$$

where the displacement $\theta$, the velocity $\omega$ and the current $i$ are the states, the voltage $v$ is the control input with the torque constant $K$ and the inductance $L$.

Although the system (1) is a third-order system and thus not mechanical system, the system (1) has a mechanical-like structure, that is, can be modeled as a port-Hamiltonian system van der Schaft (2000), Maschke \& van der Schaft (1996) with a Hamilonian $H=(1 / 2 J) p^{2}+(1 / 2) K r^{2}$

$$
\left[\begin{array}{c}
\dot{q} \\
\dot{p} \\
\dot{r}
\end{array}\right]=\underbrace{\left[\begin{array}{rrc}
0 & 1 & 0 \\
-1 & 0 & \bar{K} \\
0 & -\bar{K} & -\bar{R}
\end{array}\right]}_{A} \nabla_{x} H+\left[\begin{array}{l}
0 \\
0 \\
\bar{v}
\end{array}\right]
$$


where $x=\left[\begin{array}{lll}q & p & r\end{array}\right]^{\mathrm{T}}=\left[\begin{array}{lll}\theta & J \omega & \sqrt{L} i\end{array}\right]^{\mathrm{T}}$ is the new state, $\bar{v}=v / \sqrt{L}$ is the new input with $\bar{K}=\frac{K}{\sqrt{L}} \bar{R}=\frac{R}{L}$ and

$$
y=\nabla_{r} H
$$

is taken as the passive output. It is confirmed that this system is now passive Takegaki \& Arimoto (1981) with respect to the Hamiltonian $H$, that is,

$$
\dot{H} \leq y^{\mathrm{T}} v
$$

holds. This passive property is studied in many systems and used as a structural property to drive robust and nonliear controllers for stabilization Ortega \& Garcia-Canseco (2004) Stramigioli et al. (1998), trajectory tracking Fujimoto \& Sugie (2001) and motion generation problems Sakai \& Stramigioli (2007).

However, in this chapter, we consider a different problem, namely, impedance control problems and we do not focus on the passivity but focus on another structural property:

Lemma 1 Consider the system (1) with zero-input $v \equiv 0$ in the case of no dissipation $R=0$. Let the skew-symmetric part of the matrix $A$ be $J(x)=J(x)^{\mathrm{T}}$. Then the system has a solluton of the following PDE

$$
\nabla_{x} C(x) J(x)=0
$$

and the solution is characterized as

$$
C(x)=\bar{K} q+r .
$$

Proof This is confrimed by a direct calculation. (Q.E.D.)

This means that, in the case of no dissipation $R=0$, not only the Hamiltonian function $H$ but also the Casimir function $C$ are constant

$$
\dot{C}=0(u \equiv 0)
$$

for any the value of the Hamiltonian function $H$. Then we can express the system (1) by using the Casimir function (with respect to $J$ ) explicity.

Lemma 2 (Modeling) Consider the system (1) with zero-input $v \equiv 0$ in the case of no dissipation $R=0$. Then the coordinate transformation convert the system (1) into

$$
\left[\begin{array}{c}
\dot{q} \\
\dot{p} \\
\dot{C}
\end{array}\right]=\left[\begin{array}{rrr}
0 & 1 & 0 \\
-1 & 0 & 0 \\
0 & 0 & 0
\end{array}\right] \nabla_{x_{c}} \bar{H}+\left[\begin{array}{c}
0 \\
0 \\
\bar{v}
\end{array}\right]
$$

with the state $x_{c}=(q p C)^{\mathrm{T}}$ and the Hamiltonian function

$$
\bar{H}=\frac{p^{2}}{2 J}+\frac{(\bar{K} q)^{2}}{2}-\bar{K} q C .
$$

Proof This is also confrimed by a direct calculation although the old Hamiltonian $H$ is not equal to the new Hamiltonian $\bar{H}$. (Q.E.D.) 


\section{Exact impedance control}

In this section, we give an exact impedance control for DC motor by using Casimir functions. Proposition 1 (Main result) Consider the system (1) with the velocity input. Then the following controller

$$
\left\{\begin{array}{l}
\dot{\bar{C}}=\bar{K} q-\left(1+k_{c}\right)(\bar{K} q+r) \\
v=\bar{C} / J_{c}
\end{array}\right.
$$

converts the close-loop system into the mechanical system with the impedance parameters $J_{c}, k_{c}>0$.

Proof First we introduce an artificial Casimir function $\bar{C}$ and via the following dynamic extension

$$
\left[\begin{array}{c}
\dot{q} \\
\dot{p} \\
\dot{C} \\
\dot{\bar{C}}
\end{array}\right]=\left[\begin{array}{cccc}
0 & 1 & 0 & 0 \\
-1 & 0 & 0 & 0 \\
0 & 0 & 0 & 0 \\
0 & 0 & 0 & 0
\end{array}\right] \nabla_{x_{c}} \bar{H}+\left[\begin{array}{c}
0 \\
0 \\
v \\
\bar{v}
\end{array}\right]
$$

where $\bar{v}$ is the input corresponding to the (artificial) Casimir function.

Then the Hamiltonian function $\bar{H}$ is replaced by the following new Hamiltonian function which has a special structure suitable for impedance design with any parameters $k_{c}>0$ and $J_{c}>0$ as follows:

$$
\begin{aligned}
\bar{H}_{m e c} & =\bar{H}+\bar{K} \frac{C^{2}}{2}+\frac{\bar{C}^{2}}{2 J_{c}}+K_{c} \frac{C^{2}}{2} \\
& =\frac{p^{2}}{2 J}+\frac{\bar{K}}{2}\left(q-\frac{C}{\bar{K}}\right)^{2}+\frac{k_{c} C^{2}}{2}+\frac{\bar{C}^{2}}{2 J_{c}}
\end{aligned}
$$

due to the definition of the Casimir function. Finally the dynamic controller

$$
\left\{\begin{array}{l}
v=+\nabla_{\bar{C}} H_{m e c} \\
\bar{v}=-\nabla_{C} H_{m e c}
\end{array}\right.
$$

converts the system (1) with a dissipation $R \geq 0$ into the the following Hamiltonian system

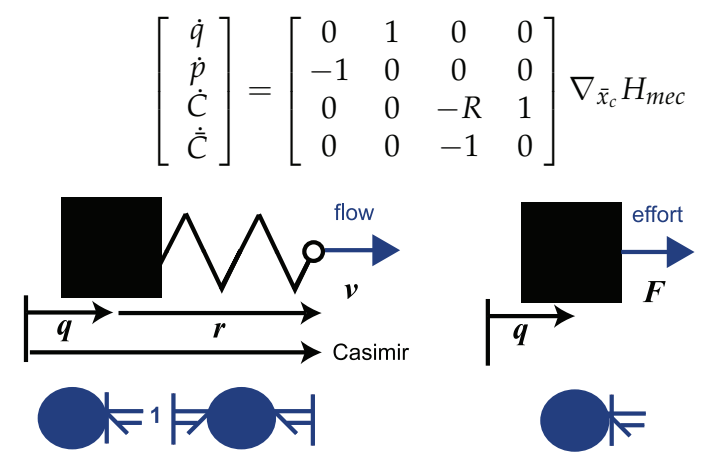

Fig. 1. A port-Hamiltonian system with flow inputs. 


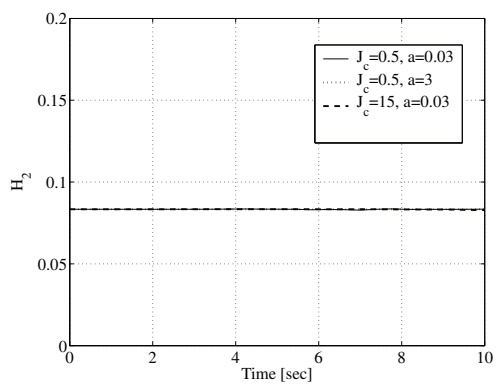

Fig. 2. Time response of $H_{m e c}$

with $\bar{x}_{\mathcal{c}}=(q p \subset \bar{C})^{\mathrm{T}}$. (Q.E.D.)

The proposed impedance control does not input the torque but the velocity, unlike the conventional impedance control for the mechanical systems. This difference is ullustrated in Fig.1. The spring coefficient $k$ between the real mass and the virtual mass is not design parameter unlike the spring coefficient $k_{c}$ between the environment and the virtual mass. Note that there is no canceling action in the controller.

\section{Numerical simulations}

Fig.2 shows the time response of the Hamiltonian function $H_{m e c}$ in the case of no dissipation (the Adams method) in the case of the parameters $J=1.5 \mathrm{~L}=0.165 \mathrm{~K}=0.47, J_{c}=0.5, a=0.03$ and the initial conditions $r(0)=q(0)=0 p(0)=0.5$. It is confrimed that the value is constant as in the acutal Hamiltonian systems

Figs.3-5 show the time responses of the Casmir function and the state $q$ and $p$ in the case of dissipation $R=3.2$. The parameters have changed $J_{c} \rightarrow 15$ and $a \rightarrow 3$.

In all cases, the nonliner behavior has changed intuitively due to the mechanial strucutre in the closed-loop system. The validity of our methods are confirmed.

\section{Conclusions}

there exist dynamics between the torque and control input and this dynamics can be dominant in certain scale. In such situation, if we neglect the dynamics or try to cancel the dynamcis, the standard impedance control can lose the stability or the control performance at least.

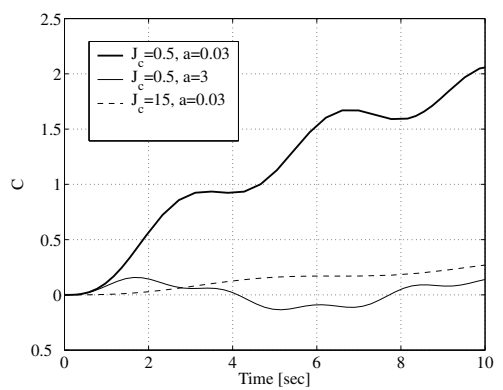

Fig. 3. Time response of $C(t)$ 


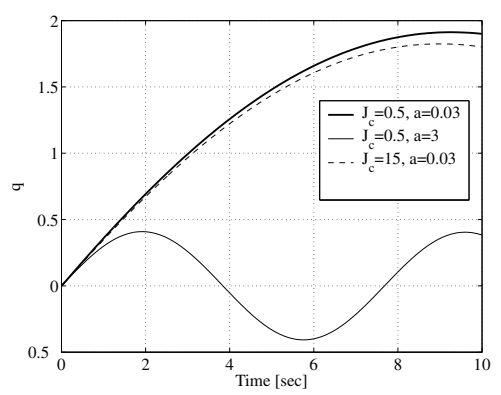

Fig. 4. Time response of $q(t)$

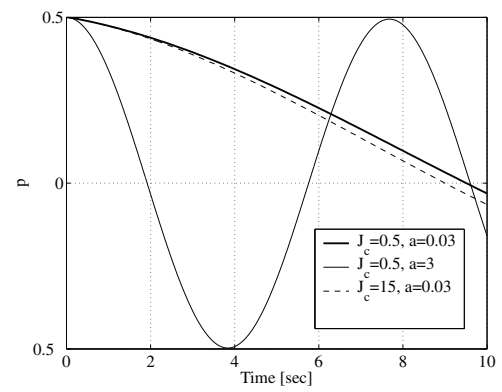

Fig. 5. Time response of $p(t)$

To overcome this problem, we need an new impedance control which takes the dynamics into account wihtout canceling any dynamics. In this chapter we give a solution for this problem by focusing on Casimir function which is rarely used in the conventional robotics.

First we give a new model of DC motor with dynamics between the torque and control input. Second, we propose a new impedance control which is based on Casimir function. Casimir function is one of the properties of port-Hamiltonian systems. Finally, we confirm the proposed method in numerical simulation.

The generalization of the proposed method and appilications to other systems (such as hydraulic systems and mascle-skelton systems) are next works in near future.

\section{Acknowledgement}

The author would like to show the appreciation to Mr. Ida Shinya for his helps in simulation works.

\section{References}

Fujimoto, K. \& Sugie, T. (2001). Canonical transformation and stabilization of generalized Hamiltonian systems, Systems \& Control Letters 42(3): 217-227.

Maschke, B. M. J. \& van der Schaft, A. J. (1996). Interconnection of systems: the network paradigm, pp. 207-212.

Ortega, R. \& Garcia-Canseco, E. (2004). Interconection and damping assignment passivity-based control: A survey, European Journal of Control pp. 1-27. 
Sakai, S. \& Stramigioli, S. (2007). Port-hamiltonian approaches to motion generations for mechanical systems, Proc. of IEEE Conference on Robotics and Automation, pp. 69-74.

Stramigioli, S., Maschke, B. M. J. \& van der Schaft, A. J. (1998). Passive output feedback and port interconnection, Proc. 4th IFAC Symp. Nonlinear Control Systems, pp. 613-618.

Takegaki, M. \& Arimoto, S. (1981). A new feedback method for dynamic control of manipulators, Trans. ASME, J. Dyn. Syst., Meas., Control 103: 119-125.

van der Schaft, A. J. (2000). L L -Gain and Passivity Techniques in Nonlinear Control, Springer-Verlag, London. 


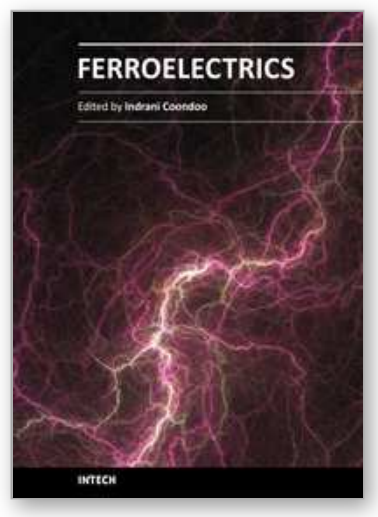

\author{
Ferroelectrics \\ Edited by Dr Indrani Coondoo
}

ISBN 978-953-307-439-9

Hard cover, 450 pages

Publisher InTech

Published online 14, December, 2010

Published in print edition December, 2010

Ferroelectric materials exhibit a wide spectrum of functional properties, including switchable polarization, piezoelectricity, high non-linear optical activity, pyroelectricity, and non-linear dielectric behaviour. These properties are crucial for application in electronic devices such as sensors, microactuators, infrared detectors, microwave phase filters and, non-volatile memories. This unique combination of properties of ferroelectric materials has attracted researchers and engineers for a long time. This book reviews a wide range of diverse topics related to the phenomenon of ferroelectricity (in the bulk as well as thin film form) and provides a forum for scientists, engineers, and students working in this field. The present book containing 24 chapters is a result of contributions of experts from international scientific community working in different aspects of ferroelectricity related to experimental and theoretical work aimed at the understanding of ferroelectricity and their utilization in devices. It provides an up-to-date insightful coverage to the recent advances in the synthesis, characterization, functional properties and potential device applications in specialized areas.

\title{
How to reference
}

In order to correctly reference this scholarly work, feel free to copy and paste the following:

Satoru Sakai (2010). An Exact Impedance Control of DC Motors Using Casimir Functions, Ferroelectrics, Dr Indrani Coondoo (Ed.), ISBN: 978-953-307-439-9, InTech, Available from:

http://www.intechopen.com/books/ferroelectrics/an-exact-impedance-control-for-dc-motor-using-casimirfunctions

\section{INTECH}

open science | open minds

\section{InTech Europe}

University Campus STeP Ri

Slavka Krautzeka 83/A

51000 Rijeka, Croatia

Phone: +385 (51) 770447

Fax: +385 (51) 686166

www.intechopen.com

\section{InTech China}

Unit 405, Office Block, Hotel Equatorial Shanghai

No.65, Yan An Road (West), Shanghai, 200040, China

中国上海市延安西路65号上海国际贵都大饭店办公楼 405 单元

Phone: +86-21-62489820

Fax: +86-21-62489821 
(C) 2010 The Author(s). Licensee IntechOpen. This chapter is distributed under the terms of the Creative Commons Attribution-NonCommercialShareAlike-3.0 License, which permits use, distribution and reproduction for non-commercial purposes, provided the original is properly cited and derivative works building on this content are distributed under the same license. 